ISSN 1991- 8690

website : http:// jsci.utq.edu.iq

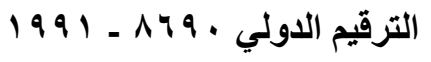

Email: utjsci@utq.edu.iq

\title{
Influence of AL Dust Particles on Air Plasma Characteristics in Cathode Sheath
}

\author{
Qusay Adnan Abbas \\ Department of Physics- Collage of Secience- University of Baghdad \\ Email: Qusayiraqi@yahoo.com.
}

\begin{abstract}
$\underline{\text { Abstract }}$
The gas discharge-dust particle interaction for a dc discharge in air with micron-sized particles is investigated. The work present illustrated the effect of AL dust particle on current and voltage of discharge and on the radial profile of plasma parameters in the cathode sheath in direct current system. The results shows, the present of dust inside the discharge increases the current while the voltage of discharge decreases. Moreover, the radial profile of plasma characteristics that measure by Langmuir probes in the presence of AL dust shows that the dust changes the plasma characteristics and affects the collective processes in such plasma system. The AL dust is charged positively in the cathode sheath and the plasma potential is negative. The presence of dust did not effect on the electron distrabution in that region.
\end{abstract}

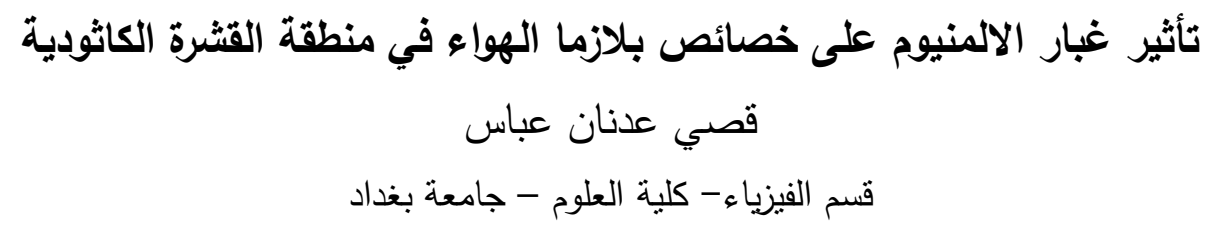

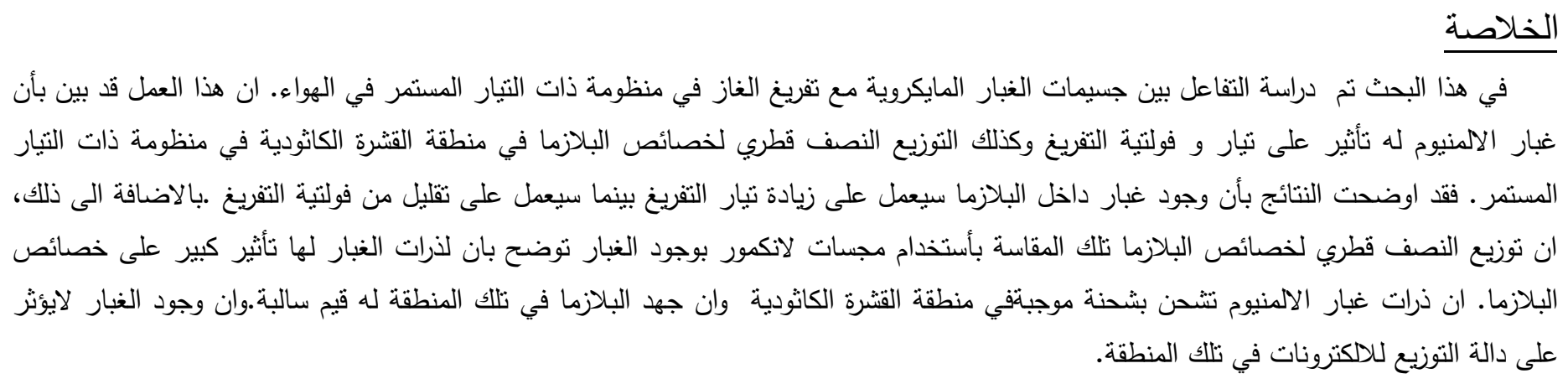

\section{Introduction}

Dusty plasma can be formed by suspending micrometresized dust particles in the low-pressure gaseous discharge. The strong negative charging ( 104 electrons per dust) on dust particles can turn the suspended dusts into the strongly coupled crystal or liquid state with sub-mm interdust spacing [1]. The dust particles can have different shapes and sizes, and they can be either perfectly insulating or perfectly conducting. It is found that in drifting plasmas, dust particles acquire an electric dipole moment. For insulating dust particles this moment is parallel to the ion drift, while for the conducting dust it is anti-parallel. For the conducting dust, the electric dipole moment is induced by the anisotropy in the potential distribution surrounding the particle and is smaller than the one for the insulating dust. In both cases, the electric dipole moment leads to a difference in the total charge on a dust particle in drifting plasmas as compared to general calculations by orbit theory for a model particle. The irregularities in the dust shape are found to influence the charge 
distribution on insulating particles but are less significant for conducting particles [2]. The study of the dusty plasma sheath is one of the important problems in the experiment equipments of plasma. A dust particle embedded in a plasma acquires an electric charge resulting from the collection of electrons and ions, the photoelectron emission, and the secondary electron emission. In laboratory plasmas where secondary emission processes due to radiation absorption and to hot particle impacts are small, the dust particle net charge is negative. An estimate of the charge is provided by assuming that an electrically floating dust particle behaves like a spherical probe with a symmetric potential. It collects thermal or monoenergetic ions, thermal plasma electrons, and in several cases, energetic electrons emitting secondary electrons [3]. Dust particles frequently appear in industrial plasmas utilized for sputtering, deposition, etching, and so on due to reactive chemical or physical processes [4]. The study of plasmas in the presence of dust is currently of great interest because of its importance for a number of applications in space and laboratory plasmas, such as magnetic confinement fusion devices, plasma processing of semiconductors, and even in electron storage rings.

\section{Experimental Arrangment}

A schematic of the plasma chamber of device that used to genetrate the dusty plasma is shown in figure (1). It consists of two aluminum electrodes of $8 \mathrm{~cm}$ in a diameter and $2 \mathrm{~cm}$ thickness. The $\mathrm{Al}$ dust particles are embedded by mechanical device into a uniform glow discharge column. This device (i.e. duster) consisted from d.c. motor which is work by applied $3 \mathrm{dc}$. volt and a circular disc (which used as a container of dust particles). The motor was work by remote control system. The disc of duster motion move two motions rotation and vibration motions. The dust particle mass which immersed into glow discharge is equal to $0.2 \mathrm{gm}$.

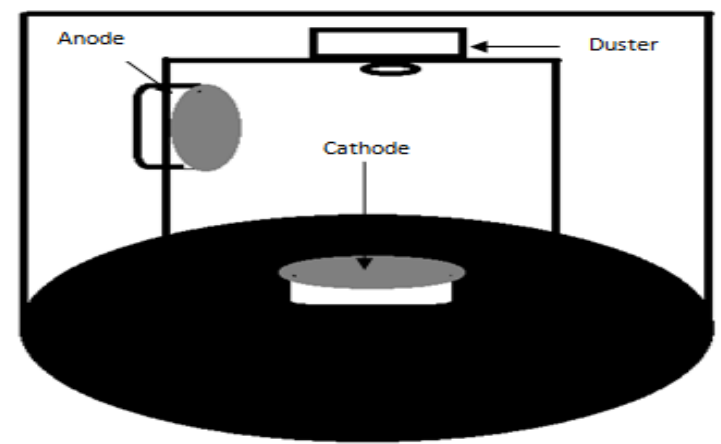

Figure (1): Diagram of the discharge chamber.
The glow discharge is formed between an anode and a cathode electrodes when a d.c. constant voltage of about $2 \mathrm{kV}$ is applied. As a results to this applied voltage, the electrical breakdown is formed in air gas at relative pressures, of about $\approx 0.1-1$ Torr. The magnetic field which used to confine the plasma particles is created by permanent behind the cathode. This field was measured by using a teslameter model magnetfeldme $\beta$ gerat. The experimental radial profile of the magnetic field along the cathode length was shown in figure (2).

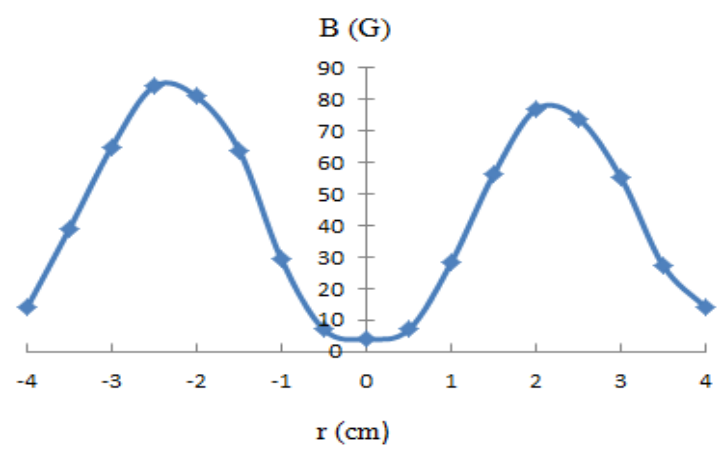

Figure (2): Radial profile of magnetic field along cathode surface.

It is clear from this figure that the radial magnetic field distrabution along cathode surface shows two peak at radial postion $2.3 \mathrm{~cm}$. Figure (3) illustrat the line of induction surrounding the cathode surface.

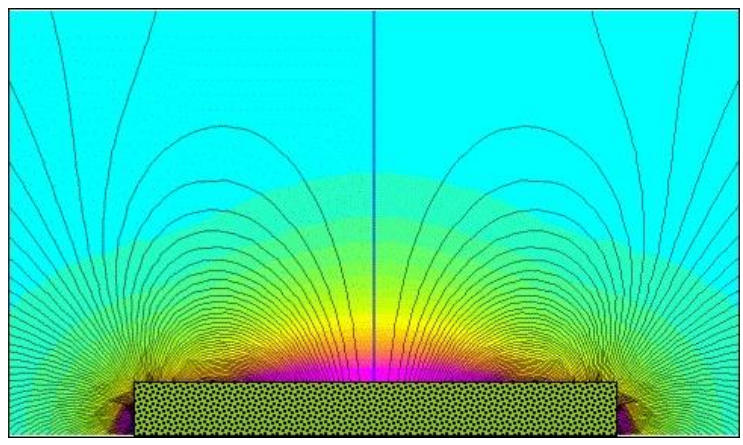

Figure (3): the magnetic field configation. 
Four cylindrical Langmuir probes located at different radial postions along the cathode surface were used to computed the radial profile of plasma parameters in cathode sheath. The probes has $3 \mathrm{~mm}$ long and $0.3 \mathrm{~mm}$ diameter, the probes tip was made from tungsten metal where the glass tube of $5 \mathrm{~mm}$ in diameter was used as external cover of probes.

\section{Evaluation of plasma parameters}

\subsection{Ion Density}

The ion current passing through an area $\mathrm{A}$ in the plasma were determined from the ion currents in the ion saturation region using the orbital motion limit (OML) probe theory. The advantage of using OML theory is that the ion density can be determined without the knowledge of the electron temperature. Here it is assumed that the plasma is isotropic, the electron temperature is much higher than the ion temperature $(\mathrm{Te}>>\mathrm{Ti})$ and the probe sheath is thick and non collisional. Assuming a maxwellian energy distribution in the unperturbed plasma, the following formula for a cylindrical probe is used to determine the ion current in the OML regime $[7,8]$ :

$\mathrm{I}_{\mathrm{i}}=\mathrm{A}_{\mathrm{p}} \mathrm{n}_{\mathrm{i}} \mathrm{e}\left(\frac{-\mathrm{eV}}{8 \mathrm{M}_{\mathrm{i}}}\right)^{1 / 2}$

where Ii , Ap, ni and Mi are ion current, probe area, ion density, and the ion mass, respectivly. By calculate the slope of the linear region (ion saturation region) of these I2 vs V curves, we can obtain an expression for the ion density.

\subsection{Electron temperature}

When the probe potential is made less negative, probe collects both ions and electrons. As the potential (probe bias) is changed further in the positive direction, the ion and electron currents collected just cancel. This current varied exponentially with probe bias voltage. This current eventually saturates at the plasma space potential value $(\mathrm{Vp})$ due to space charge limitation in current collection. In the transition region of the $\mathrm{I}-\mathrm{V}$ curve, the electron current is given by $[5,6]$ :

$I_{e}=\frac{A_{p} \cdot n_{e} \cdot e \cdot v_{t h}}{4} \cdot \exp \frac{-e V}{k T e}$.

The electron temperature can therefore be calculated directly from the I-V characteristic of the probe. The slope yields the electron temperature:
Slope $=\frac{-e}{k T e} \ldots$.

Where $T_{e}$ in $K^{\circ}$.

Slope $=\frac{1}{\mathrm{~T}_{\mathrm{e}}(\mathrm{eV})} \ldots .$.

Anyway, if the straight line of the transition region is broken into two straight lines. This fact verified that, there are two distinct Maxwellian distribution of electrons with different energies, cold and hot electrons with temperatures Tec and Teh, respectively. The slopes of these two straight segments would give the temperatures of the two groups.Consequently, the electron temperature in the case of apparent of two groups with different energy, can be estimated as [7]:

$$
\frac{1}{\mathrm{~T}_{\mathrm{e}}}=\left[\frac{n_{e c}}{n_{e}}\right] \frac{1}{T_{e c}}+\left[\frac{n_{e h}}{n_{e}}\right] \frac{1}{T_{e h}}
$$

\subsection{Electron Density}

Methods of calculating the electron density described as following: for positively biased of probe, the probe collects all the electrons and repels all the ions. The electrons current collected is nearly constant. From this current, which is called the electron-saturation current Ies, the electron density can be calculated from the following relationship[5,6]:

$$
I_{e s}=\frac{n_{e} e A_{p}}{4}\left(\frac{2 k T_{e}}{m_{e}}\right)^{1 / 2}
$$

where Te is the electron temperature and the ne is the electron number density. When there are two electron groups with different energies, then, the electron probe current is written as [7]:

$I_{\tilde{e}}(v)=I_{e c}(v)+I_{e h}(v)$

Iec and Ieh represent the cold and hot electron currents, respectively. So that, the electron density in this case is evaluated as [7]:

$n_{e}=n_{e c}+n_{e h}$

where nec and neh is the cold electron density and hot electron densities, respectively.

\subsection{Plasma Potential}

The plasma potential $(\mathrm{Vp})$ corresponds to the bias voltage where the plasma and probe are at the same potential. The plasma potential defines the potential where the electron current changes from the electron repelling current to the electron saturation current. In the "electron saturation region" electrons experience an attracting potential whereas the probe delivers a 
repelling potential to the electrons in the "electron repelling region". The potential at point of change is defined as plasma potential and can easily be obtained by looking at the rate of change of the current with respect to the applied voltage. The maximum of the first derivative $\mathrm{dv} / \mathrm{dI}$ or the zero crossing of the second derivative $\mathrm{dv} / \mathrm{dI}$ of the probe current with respect to the voltage is the way to find the plasma potential.The floating potential can be calculated from the following equation, as the bias voltage at which $\mathrm{Ii}+\mathrm{Ie}=0$.

\section{Basic Discharge Parameters}

The dc glow discharge is produced when a dc constant potential of about $2 \mathrm{kv}$ applied between two electrodes in the presence of magnetic field. Due to this external applied voltage, the discharge is formed, the electric field is established and then the electrode potential wil drop. Figures (4) and (5) shows the influence of AL dust particle on current and the voltage of discharge respectively in the presence of magnetic field. It showed be remarked from these figures, when the AL dust emmeresed into the glow discharge, the current increases while the voltage decreases. This behavior can be explined as, the presence of dust particles reduce the electron losses that occur such as classical diffusion, drift of electrons caused by $\nabla \mathrm{B} \perp \mathrm{B}$, collision of electrons with ions and neutral atom. Therefore, the discharge voltage decreases.

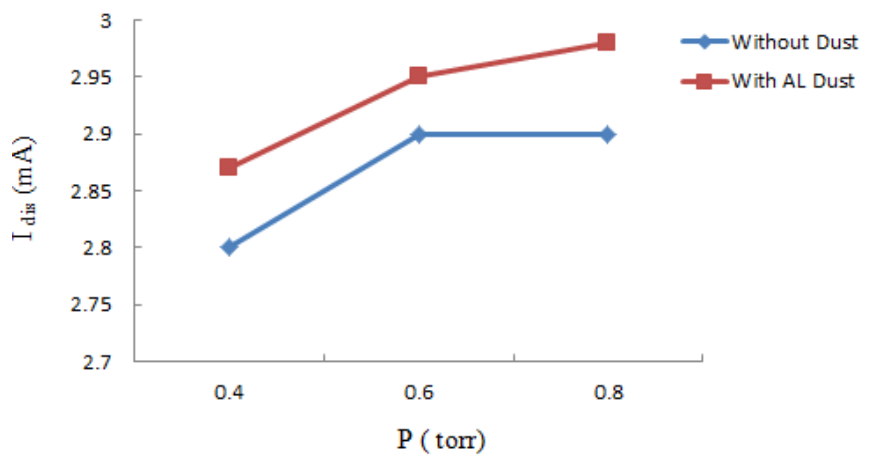

Figure (4): Schamtic the discharge current as afunction of air pressure with and without $\mathrm{Al}$ dust particles.

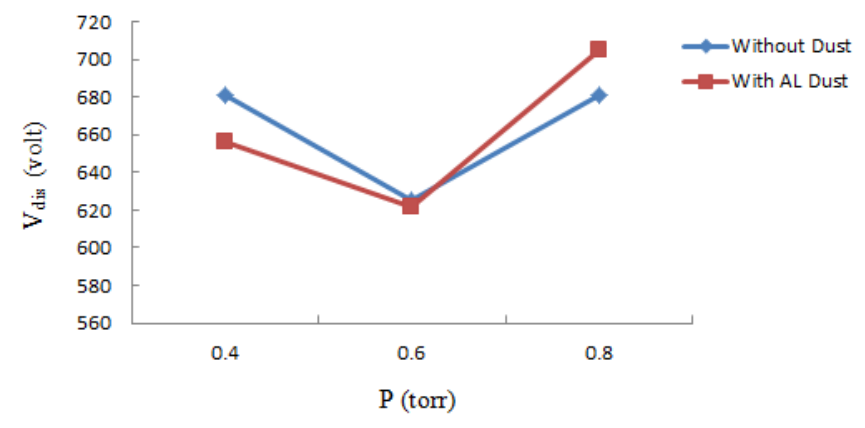

Figure (5) : Shows the voltage discharge as a function of air pressure with and without AL dust particles.

\section{Radial profile of plasma parameters}

The main diagnostic tool of the plasma characterstics consists of four Langmuir probes positioned at different radial point along the axis of cathode and at adistances is $2 \mathrm{~cm}$ from the cathode surface. Plasma parameters, namely ion density, electron density, electron temperature, and plasma potential are measured epereimentally at distance $2 \mathrm{~cm}$ from surface in the present magnetic field. Figure (6) Shows the radial profile of plasma potential with and without AL dust particle in magnetized plasma. It is explicit from this figure, the presence of dust inside the sheath causes to reduce the plasma potential toward negative values. The behavior can be explian as, when the $\mathrm{Al}$ dust immeresed in cathode sheath the electron comes towared the dust surface (because of it has high mobility), so that the dust charged negatively first. After that, the ions comes later. So that, the ion density reduce and the plasma becomes negative (i.e. plasma potential is negative). Consequently, this behavior gives an evdance to the positively charge of the dust particles in the cathode sheath region.

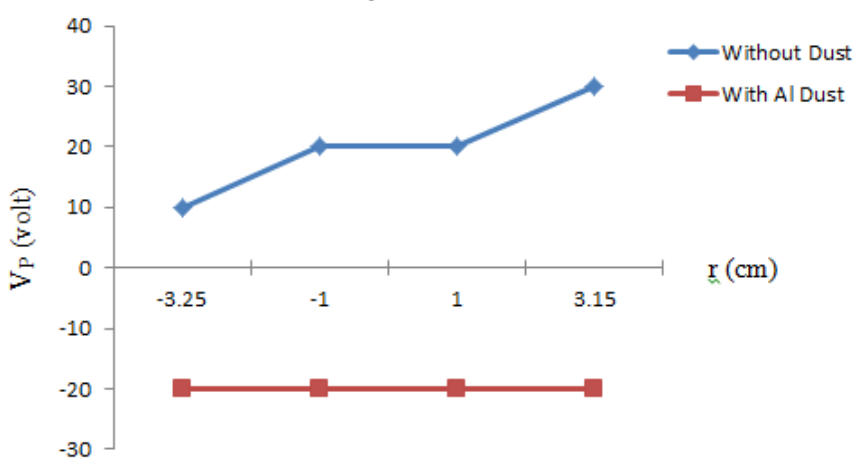

Figure (6): Radial profile of plasma potential in the presence and absence of $\mathrm{Al}$ dust particles in magnetized plasma. 
Furthermore, figure (7) illustrated the experimental data of electron saturation current (Ies) versus radial postion in the presence and absence of AL dust in magnetized plasma. As a mentioned before, the presence of dust particles in the cathode sheath reduce the losses of electrons by causing to generate anbipolar electric field. Consequently, the electrons that collected by probes will increase.

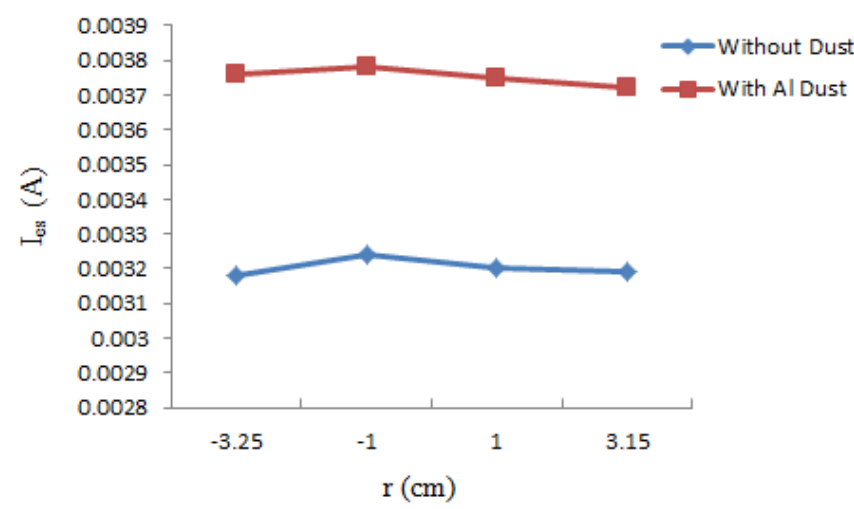

Figure (7): Radial profile of electron satration current in the presence and absence of $\mathrm{Al}$ dust particles in magnetized plasma.

According to equation (4) the cold and hot electron temperatures was calculated versus radial positions from the slope of $\mathrm{Ln}$ I-V curve of the probe characteristics. The electron temperature was calculated by using equation (5) and its radial profile is drawing in figure (8). The results shows the presence of dust reduce the electron temperature along the cathode surface. This reduction attrabuited to the fact that the presence of ambipolar electric field which cause to repelled of the electrons in the direction of probes.therefore, the electron energy decreases.

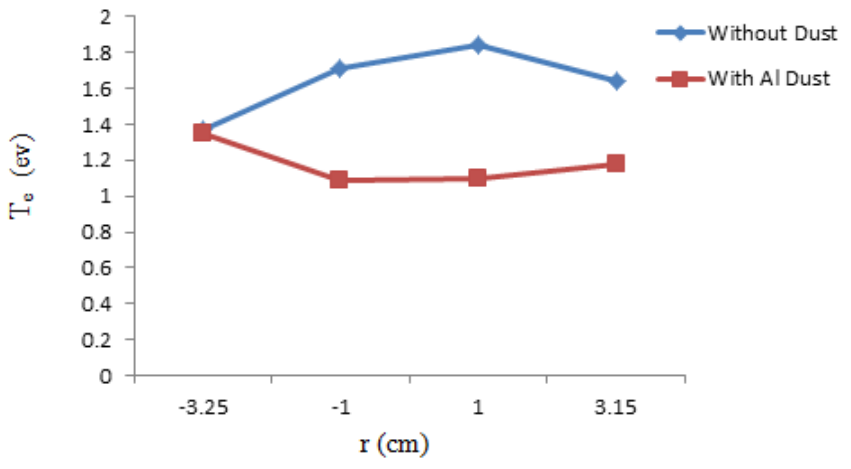

Figure (8): Radial profile of electron temperature in the presence and absence of $\mathrm{Al}$ dust particles in magnetized plasma.
In addation to the above plasma paramerers, the radial profile of electron density was determined by using equation (7) after calculated the cold and hot electron densities with experimental data of Tec and The from the experimental LnI-V curve of probes with using equation (4) ,the results is plotted in figure (9). It can explicated from this figure, the presence of dust will reduce the electron density along the cathode surface. The losses of electrons caused by collisions with other plasma particles (ions and neutral atoms) as well as electric potential of dust surface are resposible form this reduction.

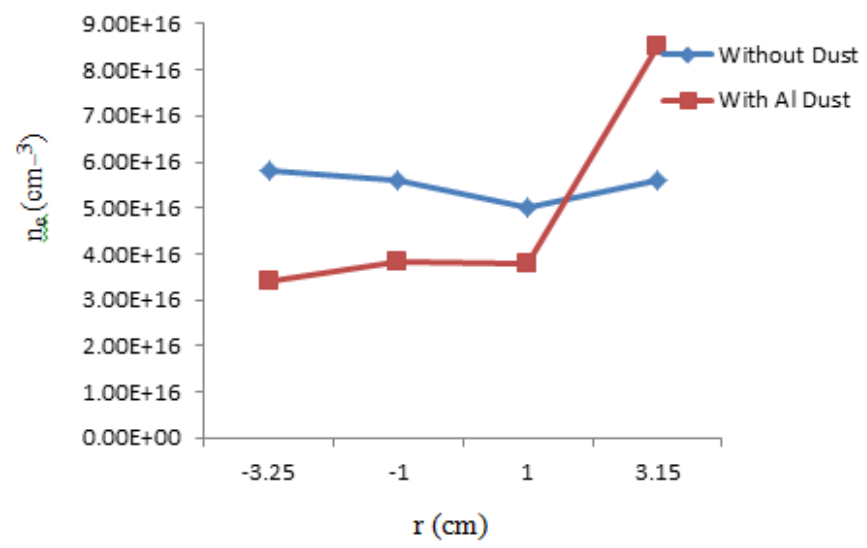

Figure (9): Radial profile of electron density in the presence and absence of $\mathrm{Al}$ dust particles in magnetized plasma.

Finally, the radial profile of ion density in the presence and absence of dust particles was calculated by using equation (1). The results was plotted in figure (10).

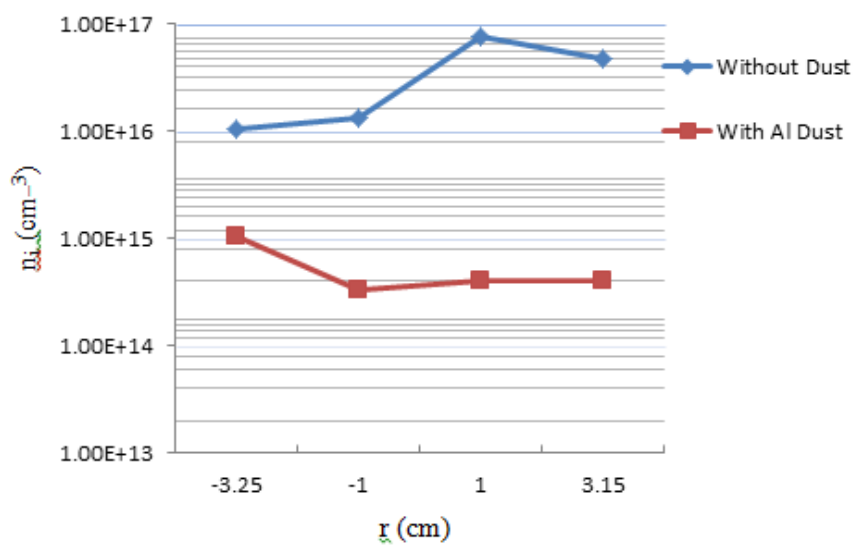

Figure (10): Radial profile of ion density in the presence and absence of $\mathrm{Al}$ dust particles in magnetized plasma. 
As mentioned above, the presence of ambipolar electric field by presence of dust particles cause to increase of ions losses by collisions as well as stike the ion on the dust particle surface. For this reason, the ion density decreases with presence of AL dust particles.

\section{Conclusion}

In the present paper, the radial profile of plasma parameters at distance $2 \mathrm{~cm}$ from the cathode surface for constant air pressure of 0.6 torr were investigated. These radial profiles illustrated that, the present of $\mathrm{AL}$ dust causes to increase the discharge current and decreases the discharge voltage. Moreover, the probe measurement of plasma parameters shows the change in the plasma characteristics in the presence of AL dust particles. The AL dust particles is charged positively in the cathode sheath. So the plasma potential in cathode sheath is negative. The presence of dust did not effect on the electron distrabution in this region.

\section{References}

1. Yen-Shuo Su, Chi Yang, Meng-Chun Chen and Lin I,"How does the supercooled dusty plasma liquid relax microscopically after quenching", Plasma Phys. Control. Fusion, 54,1, 2012.

2. B. Kakati, S. Kausik, B. Saikia, and M. Bandyopadhyay," Study on plasma parameters and dust charging in an electrostatically plugged multicusp plasma device", Physics of Plasma, 18, 063704, 2011.

3. C. Arnas, M. Mikikian, G. Bachet, and F. Doveil,"Sheath modification in the presence of dust particles", Physics of Plasmas, 7,4418,2000.

4. S. Park," Control of the charge and the nonlinear oscillation of dust particles by alternating current voltage superposition on the cathode in a direct current discharge", Physics of Plasma, 12, 062115,2005 .

5. P.Chung, L. Talbot, and K. Touryan,"Electric Probes in Stationary and Flowing Plasmas: Theory and Application",Soringer-Verlag, Berlin- Heidelbery, New York,1975.
6.R. Huddleston, and S. Leonard, "PlasmaDiagnosticTechniques", Academic Press, Inc., New York, 1965.

7. R. Merlino," Understanding Langmuir probecurrentvoltage characteristics", American J. Phys., 75, 12, 1078, 2007. 